\title{
EFECTOS DEL DÓLAR Y PETRÓLEO EN EL DESEMPLEO URBANO EN ECUADOR
}

\author{
EFFECTS OF US DOLLAR AND OIL PRICES ON ECUADOR'S URBAN \\ UNEMPLOYMENT RATE
}

\author{
Guillermo Avellán Solines, MSc. \\ Humane Escuela de Negocios \\ Instituto de Desarrollo Integral de la Persona \\ gavellan@humane.edu.ec
}

\begin{abstract}
Resumen-La recesión económica ocurrida en Ecuador durante 2016 generó un debate académico acerca del efecto de variables exógenas en el mercado laboral. El presente trabajo analiza el impacto del petróleo y el dólar estadounidense en el desempleo urbano en Ecuador entre junio de 2007 y junio 2016. Los objetivos del estudio son dos: i) presentar evidencia estadística sobre la vulnerabilidad del desempleo urbano en Ecuador ante una caída en el precio del petróleo y la apreciación del dólar; ii) realizar propuestas a nivel macroeconómico y microeconómico. La metodología aplicada es el modelo de rezagos distribuidos bajo el método de Mínimos Cuadrados Ordinarios. Las principales conclusiones de la investigación son las siguientes: i) la cotización del dólar contemporánea y el precio del petróleo con dos rezagos explican el $46 \%$ de la variación en la tasa de desempleo urbano; ii) el efecto acumulativo demuestra que una disminución de $1 \%$ en el barril de petróleo en $t-2$ y t-4 provocan en promedio un crecimiento de 2,22\% en la tasa de desempleo; iii) una apreciación de $1 \%$ en la cotización del dólar resulta en un crecimiento de 10,85\% en la tasa de desempleo.
\end{abstract}

Palabras Claves: Mercado Laboral, Variables Exógenas, Efectos Causales Dinámicos, Modelo de Rezagos Distribuidos.

Códigos JEL: J60, J63, J64, J68.

\begin{abstract}
Ecuador's 2016 recession generated an academic debate about the effect of exogenous variables in the labor market. This paper analyzes the impact of oil prices and US dollar on Ecuador's urban unemployment rate between June 2007 and June 2016. The study has two main objectives: i) to provide econometric evidence about the vulnerability of Ecuador's unemployment rate due to a drop in oil prices and appreciation of US dollar; ii) to propose policies at the macro and micro levels in order to reduce oil dependency and protect employment. The methodology employed is a Distributed Lag Model under the Ordinary Least Squares regression. The main conclusions of this study are as follows: (i) the contemporary dollar exchange rate and the oil price with two lags explain the $46 \%$ change of the urban unemployment rate in Ecuador; (ii) the cumulative effect shows that a 1\% drop in the oil price at $t-2$ and $t-4$ causes on average a 2,22\% growth in the unemployment rate; iii) a 1\% appreciation in the US dollar results in a 10,85\% growth in the unemployment rate.
\end{abstract}

Keywords: Labor Market, Dynamic Causal Effects, Exogenous Variables, Distributed Lag Model .

JEL Classification Numbers: JEL: J60, J63, J64, J68.

\section{INTRODUCCIÓN}

La economía ecuatoriana se benefició de altos precios del petróleo durante los primeros 9 años de gobierno del expresidente Rafael Correa. Sin embargo, el crudo registró dos caídas considerables durante este tiempo. Primero, desde octubre de 2008 hasta septiembre de 2009 debido a la crisis financiera en Estados Unidos; y después a partir de octubre de 2014. No obstante, es importante mencionar que en términos reales, es decir, considerando la inflación en el tiempo, los precios del petróleo ecuatoriano entre enero de 2007 y diciembre 2015 fueron superiores a los 7 años anteriores. En términos reales, el precio promedio trimestral del crudo ecuatoriano entre enero de 2007 y diciembre 2015 fue superior en 46,62\% respecto al promedio registrado entre enero de 2000 y diciembre de 2006.

Los ingresos derivados de las exportaciones petroleras fueron canalizados primordialmente a través de la inversión pública o también conocido como gasto de capital. Tanto el gasto de capital como el corriente le permitieron al gobierno convertirse en el principal motor de la economía ecuatoriana. Sin embargo, al mismo tiempo se generó una mayor vulnerabilidad ante fuerte caídas en la cotización del crudo.

El gasto de capital está financiado principalmente por créditos externos e ingresos provenientes de las exportaciones petroleras. Éstas últimas se caracterizan por ser volátiles debido a fluctuaciones en la cotización del crudo. Entre 2007 y 2016 , se pudo observar con claridad dicha volatilidad. En términos nominales, las exportaciones petroleras se ubicaron en USD 7.428 millones durante el primer año del gobierno del Presidente Correa. Tocaron su mínimo en diciembre de 2009 al llegar a USD 6.284 millones, y alcanzaron su máximo en diciembre de 2013 al ubicarse en USD 13.412 millones. Al cierre de 2016, las exportaciones petroleras registrarían su nivel más bajo en 10 años debido a la caída en el precio del crudo. De igual manera, el gasto de capital ha presentado un alto nivel de volatilidad. En el primer año del gobierno a diciembre de 2007, el

\footnotetext{
* Agradezco a Humane Escuela de Negocios, especialmente, a Marcelo Torres Bejarano y a Javier García por su respaldo incondicional en la elaboración de la presente investigación. También estoy agradecido con los economistas José Carlos Andrade Rodríguez, Alberto Dahik Garzozi, Juan Pablo Erráez Tituaña y Manuel González Astudillo por sus valiosas revisiones al documento. Cualquier error es responsabilidad del autor.
} 
gasto anual de capital comenzó en USD 2.627,5 millones y alcanzó su máximo de USD 11.812,4 millones en diciembre de 2014. También se espera una disminución significativa en este rubro al cierre de 2016.

El principal problema de financiar el gasto de capital a través de las exportaciones petroleras radica en la volatilidad del precio de crudo en los últimos 10 años. En términos nominales, la cotización del crudo ecuatoriano ha tenido un precio promedio mensual de USD 73,77/ barril entre enero 2007 y junio 2016. Sin embargo, su precio promedio máximo mensual fue de USD 117,36 en junio de 2008, mientras que su mínimo mensual llegó hasta USD 21,58/ barril en enero de 2016 . Lo anterior demuestra las fuertes fluctuaciones del precio del petróleo ecuatoriano y sus perjudiciales consecuencias para la economía en caso de tener una alta dependencia, como ha ocurrido hasta el momento.

Por otro lado, las estadísticas trimestrales sobre el desempleo urbano demuestran una relación inversa con el precio del crudo, las exportaciones petroleras y el gasto de capital. De hecho, los coeficientes de correlación entre desempleo y estas tres variables son negativos entre junio de 2007 y junio de 2016. Por ejemplo, el coeficiente de correlación entre el número de desempleados y el precio del crudo ecuatoriano es de - 0,58 durante el período analizado. Las exportaciones petroleras reflejan un coeficiente de $-0,69$; mientras que para el gasto de capital es de $-0,63$. Tal como se aprecia en los gráficos 1 y 2 , el desempleo tiende a disminuir cuando el precio del petróleo y el gasto de capital aumentan. Estos compartimientos inversos no demuestran causalidad; sin embargo, alertan la posible vulnerabilidad del mercado laboral ante una caída en el precio del crudo, debido al modelo económico del gobierno basado en los ingresos petroleros.

GRÁFICO 1: EVOLUCIÓN TRIMESTRAL DEL DESEMPLEO Y PRETRÓLEO

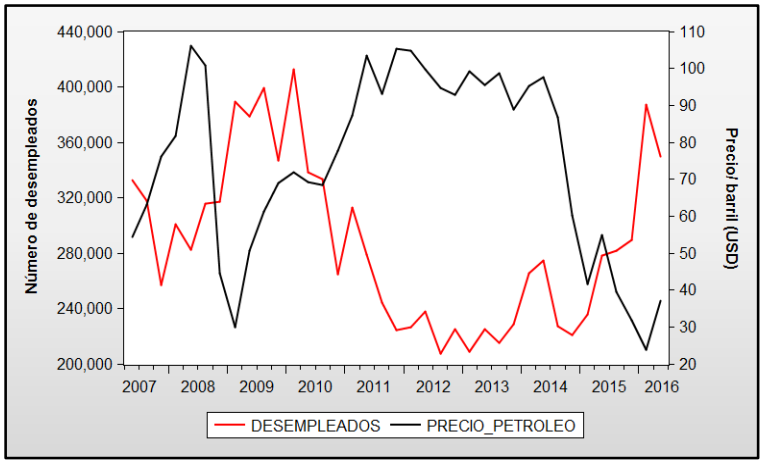

Fuente: Instituto Nacional de Estadísticas y Censos; Banco Central del Ecuador Elaboración: Autor

GRÁFICO 2: EVOLUCIÓN TRIMESTRAL DEL DESEMPLEO Y GASTO DE CAPITAL

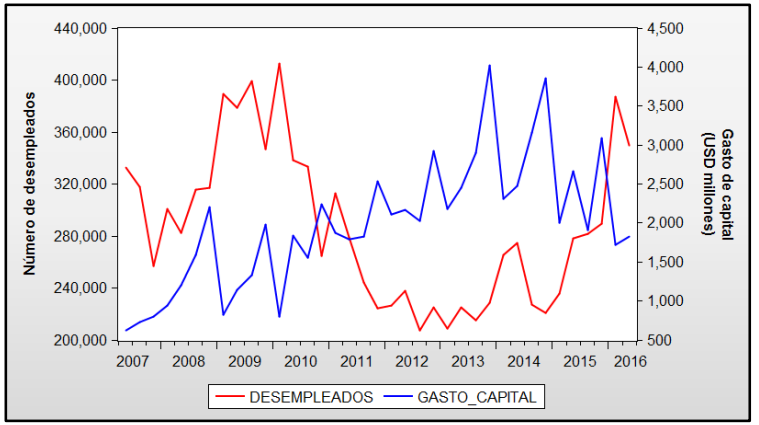

Fuente: Instituto Nacional de Estadísticas y Censos; Banco Central del Ecuador

Elaboración: Autor

Varios analistas y economistas han analizado el impacto del precio del petróleo en la economía ecuatoriana, específicamente, en la evolución del producto interno bruto (PIB). Estos estudios y análisis encuentran una alta correlación entre en el precio del crudo y la evolución del PIB no petrolero (González M., 2012). Manuel González (2012) demostró estadísticamente el efecto del precio del petróleo en la evolución del PIB no petrolero por arriba de su tendencia a partir de 2008; dicho efecto era prácticamente nulo antes del año 2005. Además, González (2015) probó un aumento de la correlación entre el precio del petróleo y el crecimiento de la economía no petrolera entre 2007 y 2012 respecto a los primeros años de la dolarización. Tanto Lucio Paredes (2010) como González (2012) alertaron de manera anticipada la vulnerabilidad de la economía ecuatoriana ante caídas en el precio del petróleo, y también destacaron la importancia de contar con fondos de ahorro para contrarrestar los efectos recesivos de una reducción en la cotización del crudo. Además, Alberto Dahik (2015) señaló al precio del petróleo como el factor detonante de la actual recesión, pero consideró al gasto público como la causa subyacente del retroceso económico. 
Otra variable económica que se encuentra fuera del control del gobierno ecuatoriano y tiene un impacto relevante en el sector externo y real es la cotización del dólar estadounidense. De acuerdo a Rafael Correa (2009), la fijación del tipo de cambio a través de la dolarización provoca consecuencias negativas en el sector externo, cuando la inflación nacional es superior respecto a la de nuestros socios comerciales o cuando estos últimos deprecian su moneda. Correa también argumenta que el tipo de cambio fijo impide al Ecuador corregir desequilibrios externos provocados por "choques exógenos", como: desastres naturales y reducciones en el precio del petróleo.

La cotización de la moneda estadounidense medida a través del Índice del dólar real (DXY) posee una correlación positiva de 0,54 con el número de desempleados entre junio de 2007 y junio 2016. Esta asociación lineal entre ambas variables demuestra que la apreciación del dólar está relacionada con un aumento del desempleo, lo cual es consistente con el argumento de Correa (2009).

\section{GRÁFICO 3: EVOLUCIÓN TRIMESTRAL DEL DESEMPLEO Y DÓLAR}

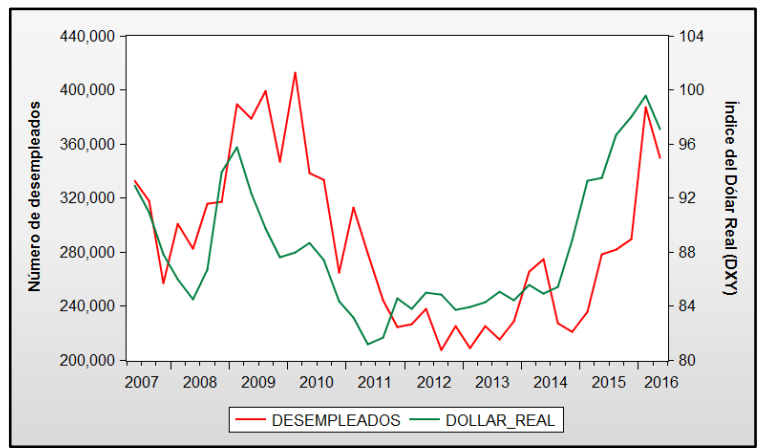

Fuente: Instituto Nacional de Estadísticas y Censos; Banco de la Reserva Federal de Saint Louis Elaboración: Autor

A pesar de las asociaciones lineales descritas previamente, se requiere utilizar métodos econométricos para demostrar causalidad. Además, es necesario utilizar la tasa desempleo en lugar del número de desempleados, para tomar en cuenta el crecimiento de la población económicamente activa en el tiempo. Sin embargo, pocas investigaciones se han enfocado en el impacto que tienen el precio del petróleo y la cotización del dólar en el mercado laboral respaldado con evidencia econométrica. Por lo tanto, el estudio busca demostrar la vulnerabilidad del desempleo urbano en Ecuador debido a la volatilidad en la cotización del crudo y dólar. De tal manera, se utiliza un modelo de rezagos distribuidos para estimar los efectos causales y acumulativos del petróleo y el dólar en la tasa de desempleo urbano en Ecuador. Específicamente, se calcula el cambio en la tasa de desempleo debido a fluctuaciones en el precio del crudo ecuatoriano y en la cotización del dólar.

La estructura del estudio será la siguiente: la sección 2 describe las fuentes y datos utilizados en la investigación. La sección 3 detalla los diferentes métodos estadísticos usados. La sección 4 muestra los resultados empíricos y la discusión de los mismos. La sección 5 menciona las implicaciones de política pública. Finalmente, la sección 6 contiene las conclusiones del trabajo.

\section{FUENTES Y DATOS}

La investigación utiliza datos trimestrales desde junio de 2007 hasta junio de 2016. Las tres variables que conforman el modelo de rezagos distribuidos son las siguientes: la tasa trimestral de desempleo en zonas urbanas; el precio promedio por trimestre del barril de petróleo ecuatoriano y la cotización promedio del dólar estadounidense por trimestre. Respecto a las fuentes de los datos, las cifras de desempleo se encuentran en la Encuesta Nacional de Empleo, Desempleo y Subempleo a junio de 2016. Mientras que los datos del precio del crudo ecuatoriano se obtuvieron del Boletín No. 1973 de Información Estadística publicado por el Banco Central del Ecuador a julio de 2016. La cotización del dólar estadounidense representada a través del índice del dólar real (DXY) se encuentra en la sección "Fred Data" del Banco de la Reserva Federal de Saint Louis durante julio de 2016.

Los precios del crudo ecuatoriano muestran una alta volatilidad, como se indica en la sección previa. De igual manera, el desempleo en el área urbana experimentó una fluctuación considerable entre junio de 2007 y junio de 2016 . Al inicio del gobierno del expresidente Correa, la tasa desempleo fue $7,5 \%$. La tasa promedio de desempleo durante estos nueve años fue 6,26\%. Su nivel mínimo fue 4,54\% en diciembre de 2015; mientras que su nivel máximo se ubicó en 9,10\% en marzo de 2010 .

Es importante mencionar que durante el período bajo análisis la población económicamente activa, con la cual se calcula la tasa de desempleo, creció en 17,91\% desde 4,44 millones en junio de 2007 hasta 5,24 millones de personas en junio de 2016.

Respecto al índice del dólar real (DXY), la cotización también registró una importante volatilidad, debido a la crisis financiera de Estados Unidos y la crisis de deuda en Europa. El dólar reportó una apreciación considerable, ya que su mínimo fue 81.16 y su máximo 99,55. Utilizando datos trimestrales de su cotización, la apreciación promedio del dólar fue 8,35\% 
durante este período. Sin embargo, al considerar fluctuación entre la cotización mínima y máxima, la apreciación llegó a $22,66 \%$.

TABLA 1: ESTADÍSTICAS DESCRIPTIVAS TRIMESTRALES

\begin{tabular}{lccc}
\hline \hline & Desempleo (\%) & Precio Petróleo (USD) & Indice Dólar (DXY) \\
\hline \hline Media & 6.26 & 74.55 & 88.24 \\
Mediana & 6.07 & 77.79 & 86.66 \\
Máximo & 9.10 & 106.09 & 99.55 \\
Minimo & 4.54 & 23.91 & 81.16 \\
Desviación Estándar & 1.37 & 24.88 & 4.95 \\
Asimetria & 0.50 & -0.47 & 0.75 \\
Kurtosis & 2.15 & 1.96 & 2.43 \\
& & 3.03 & 3.96 \\
Jarque-Bera & 2.63 & 0.22 & 0.14 \\
Probabilidad & 0.27 & 37 & 37 \\
Observaciones & 37 & & \\
\hline
\end{tabular}

Fuente: Instituto Nacional de Estadísticas y Censos; Banco Central del Ecuador; Banco de la Reserva Federal de Saint Louis Elaboración: Autor

\section{METODOLOGÍA}

La metodología aplicada en el presente trabajo es el modelo de rezagos distribuidos bajo el método de Mínimos Cuadrados Ordinarios (MCO). Dicho modelo permite estimar los efectos causales dinámicos y acumulativos provocados por una o algunas variables exógenas "Xs" sobre la variable dependiente "Y" durante un período de tiempo (Stock \& Watson, 2012). De esta manera, el modelo ayuda a calcular el impacto específico y total en la variable dependiente, dado un cambio en la variable exógena e independiente en el período actual (contemporáneo) y anteriores (rezagos).

El modelo de rezagos distribuidos de forma general es el siguiente:

$$
Y_{t}=\beta_{0}+\beta_{1} X_{t}+\beta_{2} X_{t-1}+\beta_{3} X_{t-2}+\beta_{r+1} X_{t-r}+u_{t}
$$

La forma específica del modelo que se estimará es la siguiente:

$$
\Delta Y_{t}=\beta_{0}+\beta_{1} \Delta \log \left(X_{t-2}\right)+\beta_{2} \Delta \log \left(X_{t-4}\right)+\beta_{3} \Delta \log \left(X_{t}\right)+u_{t}
$$

El modelo está compuesto por tres variables con periodicidad trimestral: la variación en la tasa de desempleo urbano es la variable dependiente $\left(\Delta Y_{t}\right)$. La primera variable independiente es la diferencia logarítmica del precio promedio del petróleo ecuatoriano con dos rezagos trimestrales $\left(\beta_{1}, \beta_{2}\right)$. Es decir, $\Delta \log \left(X_{t-2}\right)$ y $\Delta \log \left(X_{t-4}\right)$ son las primeras diferencias logarítmicas con dos y cuatro rezagos trimestrales de la misma cotización del petróleo, respectivamente. El objetivo de estos rezagos es conocer cómo el cambio en la cotización del crudo en estos dos trimestres afecta la tasa de desempleo en el período actual. De esta manera, se mide el efecto tardío que generalmente se presenta en los mercados laborales. Adicionalmente, $\beta_{3} \Delta \log \left(X_{t}\right)$ representa la diferencia logarítmica actual (contemporánea) en la cotización del índice del dólar real.

Como se mencionó previamente, cada uno de los coeficientes de la regresión MCO permiten estimar los efectos causales dinámicos o también conocidos como los multiplicadores dinámicos individuales. Por lo tanto, el multiplicador dinámico acumulativo (efecto causal acumulativo) es la suma de los multiplicadores dinámicos individuales, $\beta_{1}+\beta_{2} \ldots+\beta_{1 r+1}$, hasta el último rezago incluido en el modelo. De esta manera, se logra estimar el efecto acumulado del cambio de una unidad en la variable $\mathrm{X}$ sobre la variable $\mathrm{Y}$.

La primera transformación realizada a los datos es remover el efecto que tiene la inflación en el precio del petróleo. De lo contrario, los diferentes precios del petróleo no son comparables en el tiempo, debido al efecto que tiene el incremento de precios. Por eso, se utiliza el deflactor del Producto Interno Bruto (PIB) trimestral publicado por el Banco Central del Ecuador en sus Cuentas Nacionales. De esta manera, se remueve el efecto de la inflación en los precios del petróleo, permitiendo así que los datos se puedan comparar en el período analizado. Es importante mencionar que el índice del dólar real ya tiene descontado el efecto de la inflación, por lo que no es necesario aplicar el proceso antes mencionado a la cotización de la moneda.

La segunda transformación a los datos fue el ajuste estacional con el procedimiento X-12 a la tasa de desempleo (variable dependiente) y al precio del petróleo. Las pruebas de estacionalidad demostraron que ambas variables son estacionales, lo cual es consistente con la teoría sobre la disminución del desempleo y el aumento en el precio del petróleo en ciertos meses específicos. Este procedimiento estadístico permite corregir ese efecto en los datos. En el caso de la cotización del dólar, las pruebas de estacionalidad indicaron que esta variable no posee este problema en el período analizado.

Uno de los requisitos cuando se trabaja con series de tiempo consiste que las variables sean estacionarias, es decir, que no posean raíz unitaria. En otras palabras, la media y la varianza de las variables deben ser constantes en el tiempo. Según la prueba de Augmented Dickey-Fuller, ninguna de las variables en este modelo es estacionaria; por lo tanto, se calcula la primera diferencia para todas variables para poder trabajar con datos estacionarios. Una vez transformados los datos a primera diferencia, se volvió a aplicar la prueba de Augmented Dickey-Fuller para comprobar que las variables son estacionarias después de esta modificación.

Otra transformación importante fue el uso de logaritmos para las variables independientes, con el objetivo de medir el efecto que tienen el petróleo y el dólar en la tasa de desempleo a través de cambios porcentuales. Dado que la variable 
dependiente está en porcentaje, se puede estimar el efecto que tienen los regresores sobre la variable dependiente como elasticidades. Por esta razón, se utiliza la primera diferencia de logaritmos en las variables independientes, a fin de asegurarse que las variables sean estacionarias y facilitar el análisis de los coeficientes mediante cambios porcentuales.

Por otro lado, es necesario mencionar que las variables independientes deben ser exógenas en el modelo de rezagos distribuidos. Se entiende por variable exógena a aquella que no está bajo el control humano o también que está determinada afuera del modelo propuesto. En este caso, el precio del petróleo y la cotización del dólar son variables exógenas, ya que no están determinadas por las acciones del Estado o cualquier persona en Ecuador.

Adicionalmente, se utiliza el estimador de varianza Newey-West o también conocido como el estimador de varianza HAC por sus siglas en inglés. Este método es un estimador consistente con heteroscedasticidad y autocorrelación. El principal problema del modelo de rezagos distribuidos consiste en que pueda tener heteroscedasticidad y autocorrelación, debido a la omisión de otros determinantes que también afectan a la variable dependiente $Y_{t}$. Las variables independientes omitidas están correlacionadas en el tiempo, en consecuencia el error $u_{t}$ de la regresión también puede estar autocorrelacionado. Este problema provoca que los errores estándares de la regresión MCO no sean consistentes; y por ende, las pruebas de hipótesis y los intervalos de confianza del modelo sean erróneos. La solución consiste en estimar los errores estándares de la regresión con el estimador de varianza Newey-West (estimador HAC), para corregir la autocorrelación y heteroscedasticidad del modelo.

La fórmula del estimador de varianza HAC para $\beta_{1}$ es la siguiente (Stock \& Watson, 2012):

$$
\sigma_{\beta_{1}}^{2}=\sigma_{\beta_{1}}^{2} f_{T}
$$

$\sigma_{\beta_{1}}^{2}$, es el estimador de la varianza de $\beta_{1}$ en la ausencia de autocorrelación; mientras que $f_{T}$ es factor que ajusta la fórmula para la autocorrelación. Este factor dependerá del tamaño de la muestra "T". Si la muestra es pequeña solo necesitarán pocos autocorrelaciones, pero si la muestra es amplia se requerirá incluir más autocorrelaciones, sin embargo siempre menos que el tamaño de la muestra.

\section{RESULTADOS Y DISCUSIÓN}

A continuación se puede observar los resultados del modelo de rezagos distribuidos bajo una regresión MCO e incluyendo la corrección para los errores estándares mediante la aplicación del estimador de varianza Newey-West.

De acuerdo a los resultados de la regresión, el precio del crudo ecuatoriano con dos rezagos y la cotización del dólar contemporáneo explican el 45,6\% del comportamiento de la tasa de desempleo urbano entre junio de 2007 y junio 2016 . La constante $\left(\beta_{0}\right)$ es negativa y tiene significancia estadística al nivel de $10 \%$. El coeficiente $\beta_{1}$ nos indica que el aumento de $1 \%$ en el precio del petróleo durante dos trimestres anteriores provoca una reducción en la tasa de desempleo de $-1,35 \%$ con significancia estadística de $1 \%$. De igual manera, el coeficiente $\beta_{2}$ demuestra que el aumento de $1 \%$ en el precio del petróleo hace cuatro trimestres atrás implica una disminución en la tasa de desempleo de $-0,86 \%$ con significancia estadística cercana al $1 \%$. De esta manera, la suma de los dos coeficientes indica que el efecto acumulativo es de $-2,22 \%$. Así, el efecto acumulativo concluye que un aumento de $1 \%$ en el precio del crudo durante dos y cuatro trimestres previos provoca una reducción de la tasa de desempleo de $-2,22 \%$ en el actual trimestre. Adicionalmente, el coeficiente $\beta_{3}$ indica que un aumento o apreciación de $1 \%$ en la cotización del dólar real en el actual trimestre provoca un incremento en la tasa de desempleo de $10,85 \%$ con significancia estadística al $1 \%$. Es importante indicar que los efectos porcentuales de las variables independientes son respecto a sus primeras diferencias logarítmicas. Es decir, los cambios porcentuales no son directos sobre la variable dependiente.

MODELO DE REZAGOS DISTRIBUIDOS (MCO)

Variable dependiente: Dlog(Tasa de desempleo ajustada)

Método: Mínimos Cuadrados Ordinarios

Estimador de varianza HAC

$$
\begin{gathered}
\Delta \text { Desempleo }_{t}= \\
\underset{(0,077) \underset{(0,00)}{-0,11}-\mathbf{1}, 35 \Delta \log \left(P_{t-2}\right)}{-0,86 \Delta \log \left(P_{t-4}\right)}+\underset{(0,013)}{10,85 \Delta \log \left(D_{t}\right)+u_{t}}
\end{gathered}
$$

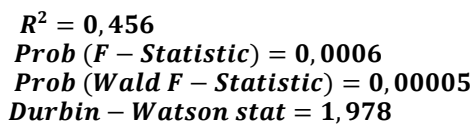

También se realizaron pruebas de autocorrelación, heteroscedasticidad y multicolinealidad. De acuerdo a estas pruebas, se descartó la existencia de estos problemas estadísticos en el modelo analizado.

Por otro lado, es relevante mencionar que la significancia de los coeficientes $\beta_{1}$ y $\beta_{2}$ con rezagos se debe al efecto tardío que generalmente ocurre en los mercados laborales. Es decir, el crecimiento de la economía provocado por el aumento en el precio del crudo se tarda cierto tiempo en convertirse en menor desempleo. Además, la apreciación del dólar posee un efecto negativo en el desempleo, debido a la pérdida de competitividad y encarecimiento de nuestras exportaciones. De esta manera, 
todos los coeficientes son consistentes con la teoría económica. También demuestran la vulnerabilidad del mercado laboral ante fuertes fluctuaciones en las cotizaciones del crudo y dólar.

Hasta el momento se ha analizado el impacto positivo que tiene el aumento del petróleo en la tasa de desempleo. No obstante, el efecto perjudicial es de la misma magnitud pero en sentido contrario. Es decir, una disminución de $1 \%$ en el precio del crudo durante dos y cuatro trimestres previos provoca un incremento de $2,22 \%$ en la tasa de desempleo durante el trimestre actual. De esta forma, las caídas en la cotización del crudo son realmente preocupantes debido al efecto nocivo en el desempleo urbano. Entre junio de 2007 y junio de 2016, se han presentado significativas reducciones en el precio del crudo durante dos trimestres consecutivos, siendo la caída acumulada más fuerte de USD 70,99 entre septiembre de 2008 y marzo 2009. Este nivel de volatilidad del crudo confirma la vulnerabilidad del mercado laboral ecuatoriano ante potenciales caídas en su precio.

Finalmente, el gráfico 4 presenta la comparación entre la serie real y la serie ajustada de la regresión. Dicho gráfico permite apreciar como el precio del petróleo y la cotización del dólar explican una proporción importante del comportamiento del desempleo urbano en el país. Existen otras variables que también afectan el comportamiento del desempleo urbano. Sin embargo, el objetivo del presente trabajo no es pronosticar el nivel desempleo; sino más bien, estimar los efectos de las variables exógenas más importantes que afectan el desempleo urbano. En este sentido, es bastante probable que el efecto del petróleo estimado en esta investigación esté subestimado debido a las variables omitidas. Es decir, el actual modelo no considera el efecto multiplicador que el precio del crudo tiene sobre otros sectores, como por ejemplo: la construcción, el consumo y el crédito.

\section{GRÁFICO 4: SERIE REAL Y SERIE AJUSTADA DE LA REGRESIÓN}

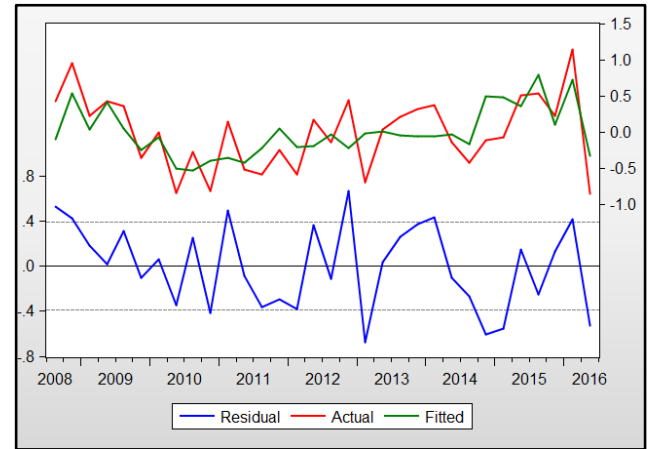

Fuente: Instituto Nacional de Estadísticas y Censos; Banco Central del Ecuador; Banco de la Reserva Federal de Saint Louis Elaboración: Autor

\section{IMPLICACIONES}

Los efectos perjudiciales que tienen la volatilidad del dólar y crudo en el desempleo urbano durante los últimos 10 años exigen la necesidad de pensar en alternativas a nivel macroeconómico y microeconómico, con el objetivo de proteger y promover el empleo en nuestro país.

En primer lugar, es de vital importancia la contratación de un seguro petrolero, que permita acordar un precio mínimo para el barril de crudo ecuatoriano durante todo el año. Esta cobertura petrolera mediante un derivado financiero ayuda a mitigar la volatilidad en el precio del crudo, evitar problemas fiscales y reducir los efectos nocivos para la economía. Los seguros petroleros generalmente tienen un alto costo, especialmente, si se pacta un precio alto. Sin embargo, el gobierno debe ser consciente de los riesgos para la economía ante una baja en la cotización del crudo.

Respecto a la cotización del dólar, los exportadores tienen la posibilidad de adquirir coberturas ante riesgos de tipo de cambio como: forwards, futuros y opciones. Mediante la contratación de estos derivados financieros tanto el sector público como privado evitan estar expuestos a pérdidas monetarias por movimientos desfavorables del dólar y petróleo.

Otra importante política macroeconómica es promover la inversión a través de asociaciones público-privadas o concesiones al sector privado. En 2015 se aprobó una nueva Ley de Incentivos de Asociaciones Público-Privadas y de Inversión Extranjera. Es necesario un mayor impulso a dicha legislación, ya que es inconcebible que el gobierno central realice todas sus obras con fondos públicos, debido a la volatilidad del precio del crudo y su fuerte impacto en los ingresos fiscales. Por otro lado, la apreciación del dólar debe convertirse en una valiosa oportunidad para innovar y mejorar la competitividad del país mediante la adquisición de bienes de capital a precios más asequibles. Para lograr esta innovación industrial, es necesario atraer nuevos capitales nacionales y extranjeros.

Adicionalmente, se requiere contar con un mayor nivel de liquidez durante períodos recesivos como los actuales. Ecuador debería contar con un fondo de estabilización que permita contrarrestar los efectos de una recesión económica en el mercado laboral. Además, dicho fondo ayudaría a tener acceso a financiamiento más barato durante recesiones, ya que el riesgo país se mantendría en niveles moderados. 
A nivel microeconómico, el gobierno impulsó el desarrollo de la pequeña y mediana empresa principalmente a través de tres legislaciones: la Ley Orgánica de Economía Popular y Solidaria (LOEPS), el Código de la Producción y la Ley Orgánica del Sistema Nacional de Contratación Pública. Estas leyes establecen una serie de incentivos y estímulos para facilitar la contratación de pequeñas empresas como proveedores del Estado para diferentes servicios y productos. Esta política pública brindó oportunidades de crecimiento para la microempresa, especialmente, aquellas ubicadas en provincias y cantones pequeños. Sin embargo, también se generó una fuerte dependencia de estos negocios pequeños a las contrataciones públicas. En consecuencia, cuando el precio del petróleo baja se afectan considerablemente los ingresos fiscales, provocando que muchos proveedores queden impagos por un tiempo prolongado o simplemente no cuentan con nuevos contratos con el Estado.

Ante las dificultades de las pequeñas empresas para encontrar oportunidades de crecimiento, la Escuela de Negocios Humane y la Fundación Acción Solidaria han propuesto un "Modelo Sostenible de Emprendimiento Social e Inclusión Laboral", que permita crear alianzas estratégicas entre el sector público, el sector corporativo y la pequeña empresa. Dicho modelo se presenta como un complemento a la Economía Popular y Solidaria propuesta por el actual gobierno, la cual se ha centrado en una visión estatal basada principalmente en la contratación pública. El objetivo de este modelo es poder fomentar que pequeños emprendimientos y asociaciones se conviertan en parte de la cadena de valor de empresas privadas, como proveedores calificados o distribuidores autorizados. El caso de éxito es el taller de confección conformado por costureras guayaquileñas, el cual se convirtió en proveedor calificado de la Tienda De Prati.

\section{CONCLUSIONES}

El modelo de rezagos distribuidos presentado en esta investigación permite estimar los efectos individuales y acumulativos que provoca un cambio en el precio del petróleo y la cotización del dólar en la tasa de desempleo urbano en Ecuador. De acuerdo al modelo econométrico presentado, la reducción de $1 \%$ en el precio del crudo durante dos y cuatro trimestres anteriores provoca en promedio un crecimiento de la tasa de desempleo de 2,22\% en el actual trimestre. Adicionalmente, la apreciación de $1 \%$ en la cotización del dólar real en el actual trimestre provoca en promedio un incremento de $10,85 \%$ en la tasa de desempleo. Ambos coeficientes tienen significancia estadística de $1 \%$.

Esta evidencia econométrica confirma los efectos causales que poseen la cotización del petróleo y el dólar en el desempleo urbano en Ecuador entre junio de 2007 y junio de 2016. Adicionalmente, la volatilidad del crudo y dólar estadounidense demuestran la vulnerabilidad del mercado laboral ante movimientos desfavorables.

La debilidad del mercado laboral ante variaciones en el precio del petróleo requiere plantearse nuevas políticas macroeconómicas como: la contratación de un seguro petrolero por parte del Estado; promover con mayor impulso la inversión privada a través de la concesión de servicios y obras públicas; y la creación de un fondo de estabilización.

En relación a los riesgos ante una fuerte apreciación del dólar estadounidense, los exportadores ecuatorianos deben considerar la adquisición de coberturas de riesgo a través de derivados financieros como: forwards, futuros, y opciones. Estos instrumentos financieros les permiten proteger el precio de sus exportaciones en caso que el dólar registre una apreciación considerable.

A nivel microeconómico es necesario implementar un modelo de emprendimiento social, como complemento a la Economía Popular y Solidaria propuesta por el gobierno. Este modelo brindará nuevas oportunidades de crecimiento para pequeños emprendimientos, y también permitirá la diversificación en las ventas de estos negocios hacia el sector privado.

\section{REFERENCIAS}

[1] Banco Central del Ecuador (2016, julio). Boletín No. 1973 de Información Estadística. Recuperado de https://www.bce.fin.ec/index.php/component/k2/item/776

[2] Correa, R. (2009). Ecuador: de Banana Republic a la No República. Bogotá, Colombia: Random House.

[3] Dahik, A. (2015). El Precio del Petróleo en el Presente y Mediano Plazo de la Economía Ecuatoriana: Consecuencias y Alternativas. Samborondón, Ecuador: Universidad Espíritu Santo.

[4] Instituto Nacional de Estadísticas y Censos (2016, julio). Encuesta Nacional de Empleo, Desempleo y Subempleo. Recuperado de http://www.ecuadorencifras.gob.ec//empleo-junio-201

[5] González, M. (4 de febrero de 2012). Relación entre el PIB no petrolero y el precio del petróleo sigue reforzándose. Recuperado de http://economiaenjeep.blogspot.com/2012/02/relacion-entre-el-pib-no-petrolero-y-el.html

[6] González, M. (8 de octubre de 2015). Correlación entre el precio del petróleo y el crecimiento. Recuperado de http://economiaenjeep.blogspot.com/2015/10/correlacion-entre-el-precio-del.html

[7] González, M. (9 de julio de 2012). Reforzamiento de la Dependencia de la Economía No Petrolera con respecto al Precio del Petróleo: ¿Desde cuándo? Recuperado de http://economiaenjeep.blogspot.com/2012/07/reforzamiento-de-la-dependencia-de-la.html

[8] González, M. (29 de octubre de 2015). La situación del empleo ha empeorado en el último año, contrario a lo que afirma el presidente. Recuperado de http://economiaenjeep.blogspot.com/2015/10/la-situacion-del-empleo-ha-empeorado-en.html

[9] Lucio-Paredes, P. (2010). Ecuador: de la No República... a la No República. Quito, Ecuador: Trama editorial.

[10] Stock, J., Watson, M. (2012). Introduction to Time Series regression and Forecasting. Introduction to Econometrics (pp. 558-624). Harlow, Inglaterra: Pearson.

[11] Stock, J., Watson, M. (2012). Estimation of Dynamic Causal Effects. Introduction to Econometrics (pp. 625-672). Harlow, Inglaterra: Pearson. 